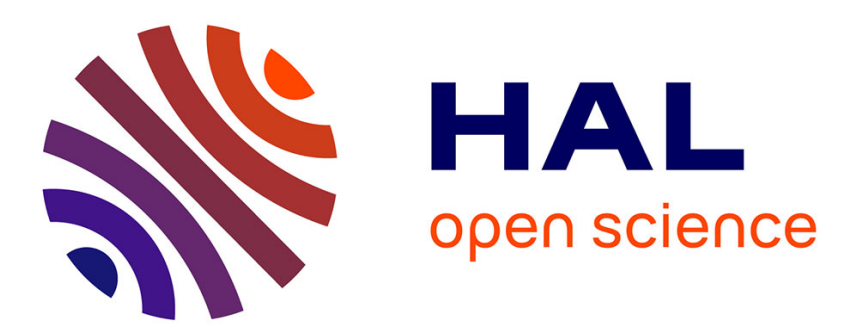

\title{
Magnetorelaxometry using Improved Giant MagnetoResistance Magnetometer
}

Matthieu Denoual, Sébastien Saez, F. Kauffmann, Christophe Dolabdjian

\section{To cite this version:}

Matthieu Denoual, Sébastien Saez, F. Kauffmann, Christophe Dolabdjian. Magnetorelaxometry using Improved Giant MagnetoResistance Magnetometer. Sensors and Actuators A: Physical , 2010, 159 (2), p184-188. 10.1016/j.sna.2010.03.029 . hal-00980139

\section{HAL Id: hal-00980139 https://hal.science/hal-00980139}

Submitted on 17 Apr 2014

HAL is a multi-disciplinary open access archive for the deposit and dissemination of scientific research documents, whether they are published or not. The documents may come from teaching and research institutions in France or abroad, or from public or private research centers.
L'archive ouverte pluridisciplinaire HAL, est destinée au dépôt et à la diffusion de documents scientifiques de niveau recherche, publiés ou non, émanant des établissements d'enseignement et de recherche français ou étrangers, des laboratoires publics ou privés. 


\title{
Magnetorelaxometry using Improved Giant MagnetoResistance Magnetometer
}

\author{
M. Denoual ${ }^{\mathrm{a}, *}$, S. Saez ${ }^{\mathrm{a}}$, F. Kauffman ${ }^{\mathrm{b}}$, C. Dolabdjian ${ }^{\mathrm{a}}$ \\ a Groupe de Recherche en Informatique, Image, Automatique et Instrumentation de Caen (GREYC), CNRS UMR 6072 - ENSICAEN and Université de Caen Basse Normandie, \\ 6 Bd. du Maréchal Juin, 14050 Caen Cedex, France \\ ${ }^{\mathrm{b}}$ Laboratoire de Mathématique Nicolas Oresme (GREYC), CNRS UMR 6119 - Université de Caen Basse Normandie, BP 5186, F 14032 Caen Cedex, France
}

\begin{abstract}
A B S T R A C T
Magnetorelaxometry measurements on immobilized superparamagnetic magnetite $\left(\mathrm{Fe}_{3} \mathrm{O}_{4}\right)$ nanoparticles in freeze-dried samples using a low-cost Improved Giant MagnetoResistance Magnetometer (IGMRM) are presented. Fits to relaxation phenomenological model based on Moment Superposition Model (MSM) are used to extract the characteristic values. Concentrations of iron down to $3.85 \mu \mathrm{mol}$ are identified in a $150 \mu \mathrm{l}$ volume from experimental measurements. Also, the extracted amplitude characteristic value confidence intervals associated to measurements and detection performances are given.
\end{abstract}

\section{Introduction}

Magnetic NanoParticles (MNPs) have many applications in the biomedical field ranging from biomaterial manipulation and drug delivery in targeted therapy [1-3] to such medical applications as treatment through magnetic heating [4]. MNPs used as labels for biosensing have several potentials advantages over other ones. As labels, MNPs have a low-toxicity [5] and allow detection in opaque media [6]. Making use of these advantages, magnetic immunoassays have been developed to quantify biological targets. The so-called MAgnetic Relaxation ImmunoAssay (MARIA) is based on the magnetic relaxation of magnetic nanoparticles [7]. The Magnetorelaxation technique, first proposed 14 years ago, has since been widely developed and used [8-10].

The first magnetorelaxometry measurements were carried out using SQUID sensors [11]. The SQUID is known to be one of the most sensitive solid state magnetic field magnetometers, but it requires liquid helium or liquid nitrogen cooling and is limited in terms of picking up the initial part of the relaxation cycle. In recent years, a similar system using room temperature fluxgate magnetometers have been developed [8]. These can be put closer to the sample than SQUID, partially compensating for their lower intrinsic sensitivity and are able to pick up the entire relaxation cycle. Recent magnetorelaxometry experiments with SQUID and fluxgate experimental set-ups [12] have shown that sensed field were not, up to a point, influenced by the experimental set-up.

The Giant MagnetoResistive (GMR) effect $[13,14]$ has been proposed for biosensing $[15,16]$ and investigated with a view to replace

\footnotetext{
* Corresponding author.

E-mail address: mdenoual@greyc.ensicaen.fr (M. Denoual).
}

SQUIDs as magnetic sensors for the detection of MNPs [17]. The noise performances of GMR sensors are worse than those of SQUIDs but this can be compensated by standoff reduction in the case of GMR sensors and it leads to comparable signal-to-noise ratio when magnetic microparticle detection is considered [17].

This paper deals with the potential use of a low-cost IGMRM $[18,19]$ to perform magnetorelaxometry measurements on magnetic nanoparticle diluted samples. We first present the characteristics of the IGMRM and its implementation in the magnetorelaxometry measurement set-up. Then, we present IGMRM capability to observe magnetic nanoparticle relaxation versus time. Finally, we introduce a model to evaluate the confidence interval of the extracted characteristic value from the fits and the intrinsic set-up noise.

\section{Principle of operation}

Magnetorelaxometry technique is based on the measurement of magnetic signal induced by superparamagnetic nanoparticles under an external applied magnetic induction. When the magnetic induction is turned on, the magnetic moments of the nanoparticles are aligned and sample shows measurable signals ( $c f$. Fig. 1) which are proportional to nanoparticle magnetization, $m_{M N P S}$. Their amplitudes depend on the applied external magnetic induction, the MNP properties, MNP distance from the magnetometer and MNP concentration. When the external magnetic induction is suddenly turned off, the magnetic moments of the MNP sample relax according to different mechanisms, depending upon whether the nanoparticles are mobile or immobilized [20]. For immobilized MNPs, the magnetization decays only via the Néel mechanism [8]. Mobile MNPs also relax through Brownian motion. Models have been proposed to express the relaxation phenomenon of ensembles 


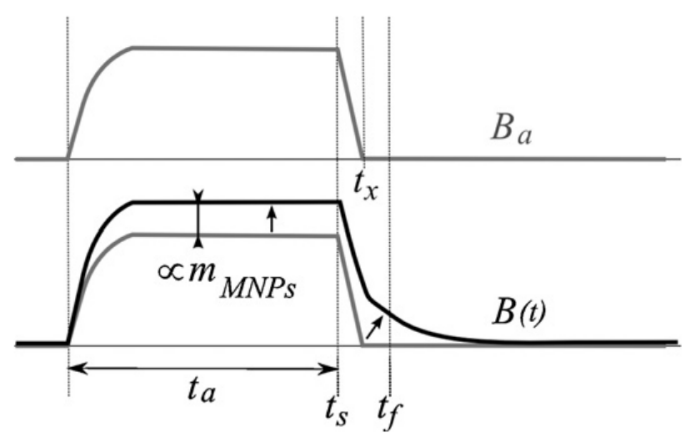

Fig. 1. Typical MNP signal response. External applied magnetic induction, $B_{a}$, and sensed magnetic signal, $B(t)$, in magnetorelaxometry experiments. $t_{f}$ is the start recording time. Beyond, data points are used to fit curves with the relaxation phenomenological expression. $t_{S}$ corresponds to the cut-off time of the magnetic induction. $t_{x}$ is the magnetic induction falling time.

of MNPs, the so-called Moment Superposition Model (MSM) [21]. By measuring the magnetization decay, MNPs can be distinguished by their different relaxation behavior, whether they are bound or not. Then, they can be used as biomarkers when functionalized with specific biomaterials, such as antibodies or antigens.

\section{Experimental set-up}

Magnetorelaxometry measurements were performed on a series of five $150 \mu \mathrm{l}$ samples of dilute magnetite $\left(\mathrm{Fe}_{3} \mathrm{O}_{4}\right)$ superparamagnetic MNPs. The MNPs are commercially available ones from Chemicell $\mathrm{Gmbh}$ and exhibit diameter core size around $10 \mathrm{~nm}$. Each new sample is diluted by a factor of two with deionized water from the previous one, from $\mathrm{N}_{1}$, being the most concentrated with a Fe content of $C_{N 1}=22.9 \mathrm{mg} / \mathrm{ml}$, to $N_{5}$, the least concentrated (diluted by a factor of 16 ). The MNPs were immobilized by freezedrying, so that the sample magnetization, $m_{M N P s}$, decays via the Néel mechanism and an associated characteristic phenomelogical time constant, $\tau_{\text {immobilized }}$ derived within the MSM framework.

The experimental set-up (cf. Fig. 2) is similar to the fluxgate magnetorelaxometry system developed by Ludwig et al. [22]. The IGMRM, few $\mathrm{mm}^{3}$, is located near the center of a Helmholtz coil with its sensitive axis perpendicular to the external applied mag- netic induction in order to minimize the signal in the absence of sample. Samples containing the magnetic nanoparticles are positioned close to one end of the IGMRM, in practice $(\approx 3 \mathrm{~mm})$, so that the signal is maximized. The Helmholtz coil produces a magnetic induction, $B_{a}$, during a time interval, $t_{a}$. The magnetic induction tend to align the magnetic moments of the MNPs because of magnetic force giving rise to a torque, leading to a rotation of the magnetic moment along its axis. This alignment mechanism overcomes the magnetic MNP moment agitation due to thermal energy when a sufficiently high field is applied (the ratio of aligned particles can be quantified by the Langevin function). After switching-off the external applied magnetic induction, random scattering induces a small relaxation of the sensed magnetic signal. For the relaxation processes to be observable, the magnetic induction must be switched off abruptly. For this purpose, a voltage amplifier and drive electronics, providing a linear decay of the magnetic induction within $400 \mu \mathrm{s}$, were built. The current flowing through the Helmholtz coil, $I_{\text {coil }}$, was determined using a resistor placed in series. The signal was low-pass filtered $(10 \mathrm{kHz})$, amplified, and averaged (128 times) to improve the signal-to-noise-ratio. All measurements were performed at room temperature within the GREYC magnetic shielded room (one layer in soft iron, four in $\mu$ metal and one in aluminum, with an internal white noise lower than $20 \mathrm{fT} / \sqrt{ } / \mathrm{Hz}$ ).

The IGMRM $[18,19]$ uses analog and low field magnetoresistor (MR) sensor AA002 from the NVE Corporation [23]. Sensor consists of unshielded and shielded sensitive elements arranged in a bridgecircuit configuration. The latter was supplied by a voltage of $12 \mathrm{~V}$. The IGMRM readout electronic is made, similarly, as classical magnetometers and operates in optimal field locked loop mode. The latter minimizes the bridge-circuit non-linearity and hysteresis. The design of the feedback circuit was made with a single Operational Amplifier and two feedback elements (coil and resistor). In the loop, a bias voltage determines the bias field. It places the sensor at its highest sensitivity. Moreover, the bias field is low affected by the bias voltage fluctuations which are filtered. In the bandwidth, the flat frequency response of the closed-loop sensitivity is determined by the geometrical factors of the feedback coil and a resistor. To summarized, the IGMRM yielded an intrinsic magnetic noise spectral density of $50 \mathrm{pT} / \sqrt{ } \mathrm{Hz}$ (equivalent to intrinsic GMR noise) at frequencies higher than $4 \mathrm{kHz}$. The measured values for sensitiv-

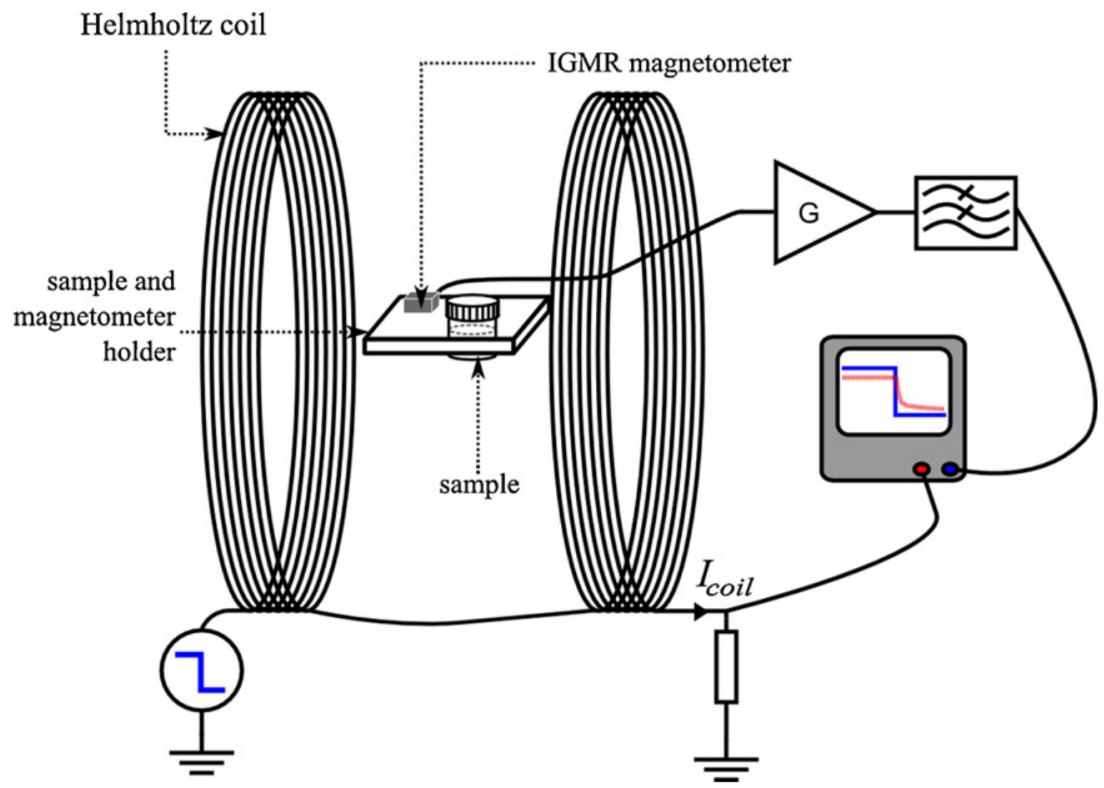

Fig. 2. Experimental set-up for magnetorelaxometry experiments. 


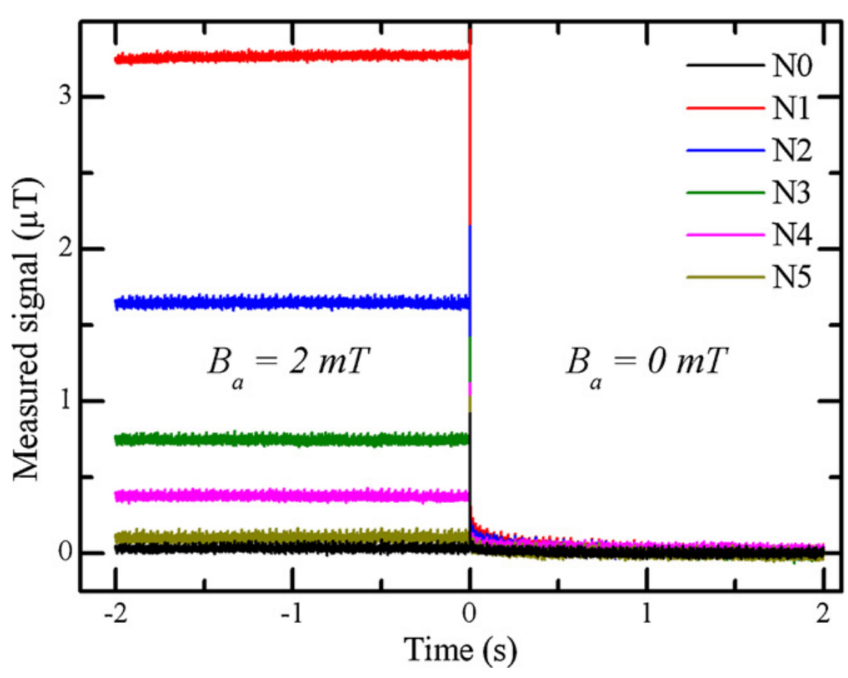

Fig. 3. Averaged (128 times) magnetic relaxation signal of freeze-dried magnetite samples, sensed with an IGMRM. $\mathrm{N}_{1}$ and $\mathrm{N}_{0}$ correspond to the most concentrated sample and to the blank measurement, respectively. To account for offset drifts of the system, each individual curves were offset-corrected to zero at $t=2 \mathrm{~s}$.

ity, band-width and dynamic range were $1 \mathrm{kV} / \mathrm{T}$, more than $300 \mathrm{kHz}$ and $120 \mathrm{~dB} / \sqrt{ } \mathrm{Hz}$, respectively. The slew-rate was measured to be over than $37 \mathrm{~T} / \mathrm{s}$.

\section{Results}

\subsection{Measured signals and measured field amplitudes versus the sample concentration in nanoparticles}

The measured signals were averaged 128 times to produce curves, which are used in the experiments for different concentrated freeze-dried magnetic nanoparticle samples, as given in Fig. 3. The samples were magnetized in a magnetic induction, $B_{a}$, of $2 \mathrm{mT}$ during $2.5 \mathrm{~s}$ every $5 \mathrm{~s}$. A non-zero response without sample, during blank measurement, likely due to the non-orthogonality between the Helmholtz coil and IGMRM axes, was observed, initially. To compensate for this misalignment and for the equivalent magnetic field drift of the measuring system, we notice that the system was offset corrected. Indeed, measurements on each sample were consistently preceded by a blank measurement that was subsequently subtracted to the measurements. The difference between each pair of results yielded a signal proportional to the MNPs magnetization, $\propto m_{M N P S}$, of each sample. Fig. 4 shows the measured stray field of the magnetized samples as a function of their concentration. The signals exhibit near linear variation with sample concentration, as expected.

\subsection{Magnetorelaxometry and amplitude extracted}

The signal decay caused by Néel relaxation mechanism of the ensemble of immobilized MNPs, when the external magnetic induction is turned off, could be clearly observed for the most concentrated samples, $\mathrm{N}_{1}$ and $\mathrm{N}_{2}$, as shown in Fig. 5. Similarly to previous curves, a blank measurement was subtracted from measurement with sample. After the initial transition, the experimental sensed relaxation curves of immobilized superparamagnetic MNPs can be fitted by the expression [22]

$$
\begin{aligned}
B(t) \approx & \kappa\left[\left.B_{\text {off immobilized }}\right|_{N_{i}}+\left.B_{\text {immobilized }}\right|_{N_{i}} \ln \left(1+\frac{\tau_{\text {immobilized }}}{t-t_{0}}\right)\right] \\
& +B_{o f f}+b_{n}(t)
\end{aligned}
$$

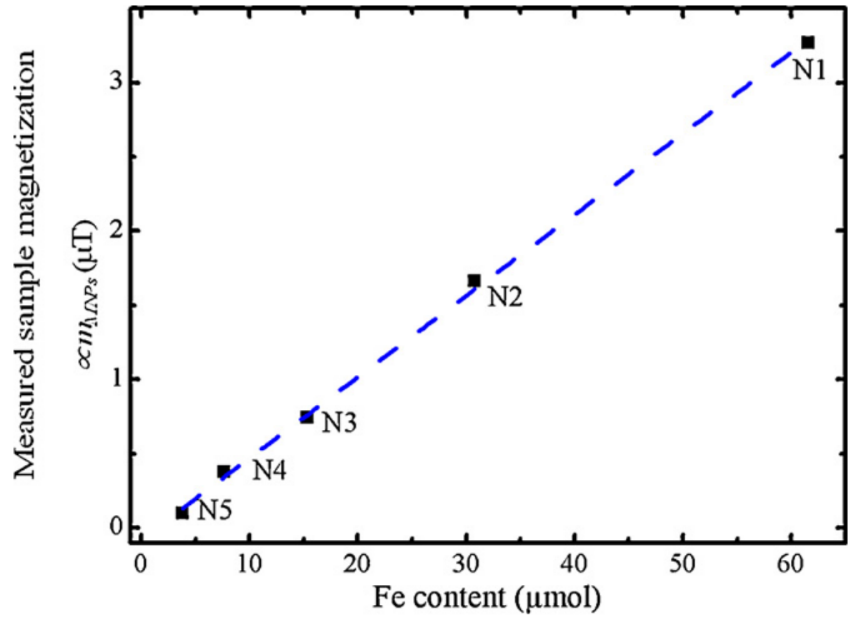

Fig. 4. Measured and averaged (128 times) sample magnetization, proportional to $m_{M N P s}$, versus MNP freeze-dried sample concentration with associated linear regression (dotted line, $\propto m_{M N P s}=-0.076+0.055 \cdot C_{N_{i}}$ ).

where $\kappa$ is a coefficient depending on the position or distance of sample to IGMRM and on the spatial magnetometer resolution [24]. $b_{n}(t)$ is the set-up noise and $B_{\text {off }}$ stands for the offset of the IGMRM. $\left.\kappa B_{\text {off immobilized }}\right|_{N_{i}},\left.\kappa B_{\text {immobilized }}\right|_{N_{i}}$, and $\tau_{\text {immobilized }}$ are fit parameters. $t_{0}$ corresponds to the beginning of the Néel relaxation mechanism after the switch-off at time $t_{s}$ of the applied magnetic induction, $B_{a}$. For the fitting procedure, considering the sampling period and the electronic set-up, the start recording time was fixed at $t_{f}$ so that $t_{f}-t_{x}=800 \mu \mathrm{s}$. The value of $\tau_{\text {immobilized }}$ and $t_{0}$ were determined with the fit of the most concentrated sample, $\mathrm{N}_{1}$. Theses two parameters are used as a constant to fit $\mathrm{N}_{2}$ to $\mathrm{N}_{5}$ sample curves. Fig. 6 illustrates the $\mathrm{N}_{1}$ sample experimental relaxation curve and its associated fit. The magnetic field amplitude image, $\left.\kappa B_{\text {immobilized }}\right|_{N_{i}}$, extracted from fits, is plotted versus sample concentrations ( $c f$. Fig. 7). Also, we observe a correlation between the extracted parameter value, $\left.\kappa B_{\text {immobilized }}\right|_{N_{i}}$, versus MNP concentration of samples.

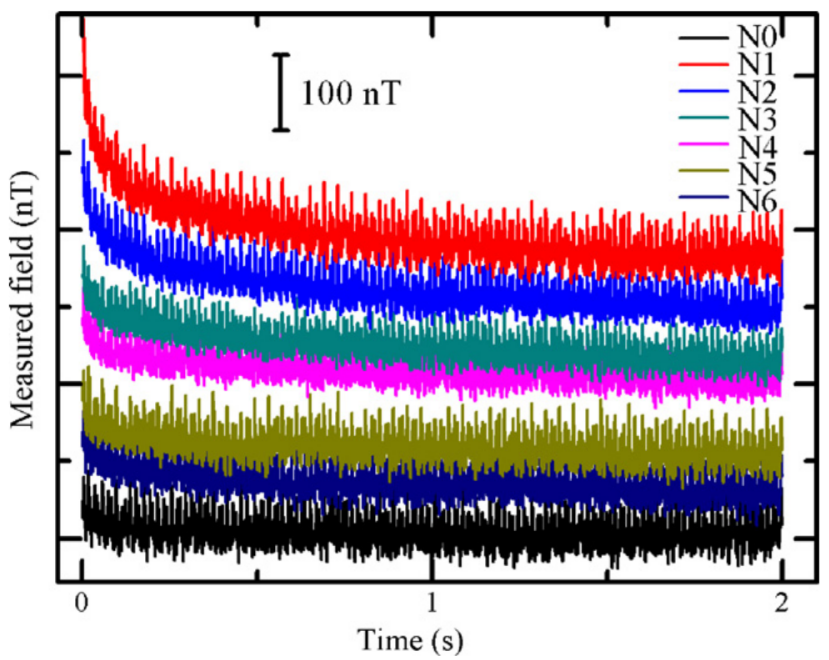

Fig. 5. Close view, in the transition region, of the magnetic relaxation signals of the freeze-dried magnetite samples (averaged 128 times). Curves are presented in a waterfall plot. $\mathrm{N}_{1}$ and $\mathrm{N}_{0}$ correspond to the most concentrated sample and to the blank measurement, respectively. 


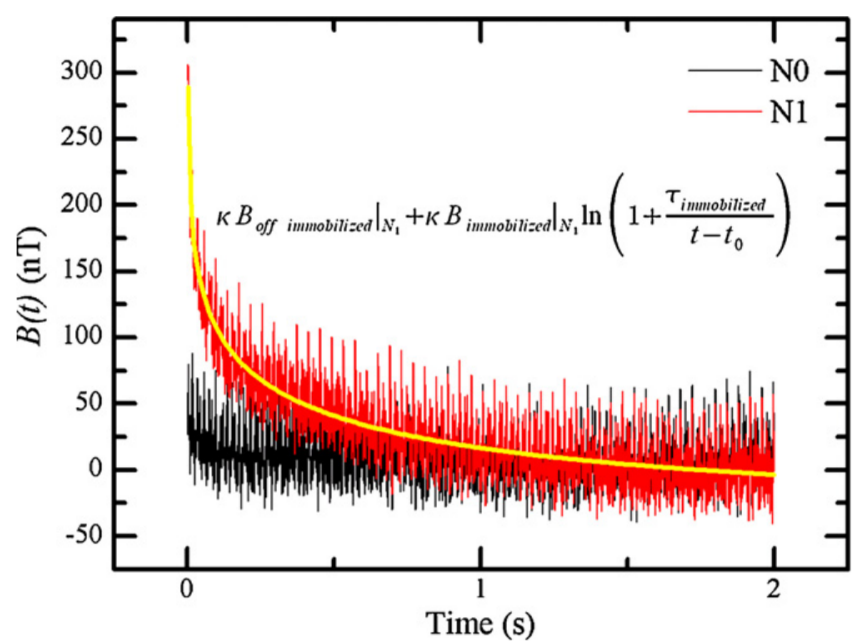

Fig. 6. Magnetic relaxation signal of the most concentrated freeze-dried sample $\mathrm{N}_{1}$ with its associated fit to the expression of relaxation (1). $\mathrm{N}_{0}$ corresponds to the blank measurement. ( $\left.\kappa B_{\text {off immobilized }}\right|_{N_{1}}=-42 \mathrm{nT} ;\left.\kappa B_{\text {immobilized }}\right|_{N_{1}}=47 \mathrm{nT}$; $\tau_{\text {immobilized }}=2.7 \mathrm{~s}$ ).

\subsection{Confidence interval of extracted amplitude}

In this paragraph, we evaluate the confidence interval of magnetic field amplitude extracted from fits. As mentioned previously, the fit to the MSM phenomenological relaxation mechanism model was done in two consecutive steps. Firstly, the values of $\tau_{\text {immobilized }}$ and $t_{0}$ were determined with the help of the NELDER-Mead method [25] in the most concentrated sample's case, $\mathrm{N}_{1} . \tau_{\text {immobilized }}$ of $2.7 \mathrm{~s}$ extracted here is in good agreement with the MSM model according to which this quantity is for small fields of the order of the magnetization time [21] (2.5 s here). Secondly, those two parameters were kept constant for further fitting. Considering this, MNP relaxation can be seen as a linear model in $\left.\kappa B_{\text {off immobilized }}\right|_{N_{i}}$ and $\kappa B_{\text {immobilized }} \mid N_{i}$, if $\tau_{\text {immobilized }}$ and $t_{0}$ are known. Also, (1) is simplified by defining $\tau(t)$ as follow

$\tau(t)=\ln \left(1+\frac{\tau_{\text {immobilized }}}{t-t_{0}}\right)$

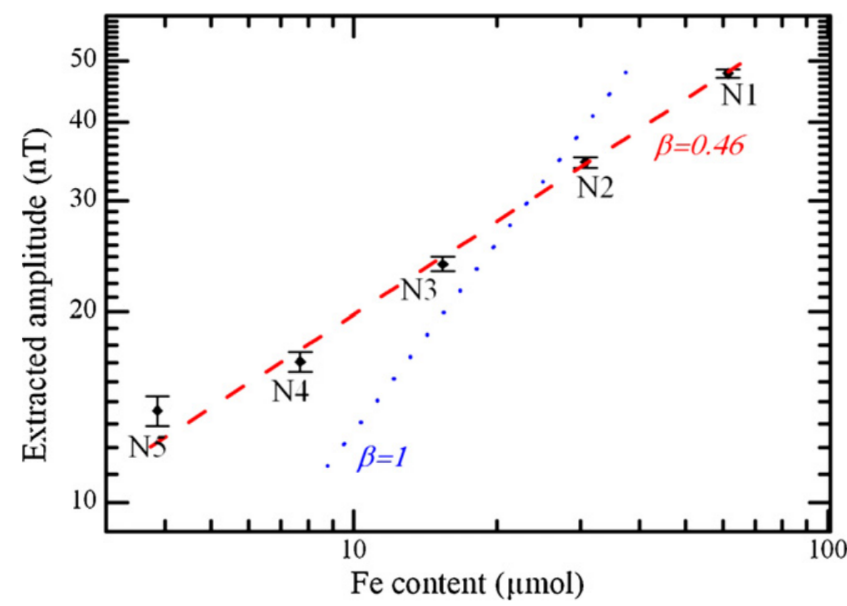

Fig. 7. $\kappa B_{\text {immobilized }} \mid N_{i}$ amplitude obtained from fitting using least mean square regression on the measured relaxation curves with (1) versus iron freeze-dried sample concentration. The $99 \%$ confidence interval was calculated with (4) after evaluation for each sample of the magnetic relaxation signal noise variance in the respect of the considered sample. Noises are obtained by differentiation between the measured relaxation curves and the curves extract from fit. The dashed line illustrates the slope $(\beta=0.46)$ obtained for the measurements. In theory, a linear relationship $(\beta=1)$, illustrated by the dotted line, should be expected between the amplitude, $\left.\kappa B_{\text {immobilized }}\right|_{N_{i}}$, and MNP concentration, $C_{N_{i}}$.
Consequently, experimental sampled curves can be rewritten as

$$
\begin{aligned}
B_{i} \approx \kappa & {\left[\left.B_{\text {off immobilized }}\right|_{N_{i}}+\left.B_{\text {immobilized }}\right|_{N_{i}} \cdot \tau\left(i T_{S}\right)\right] } \\
& +b_{n}\left(i T_{S}\right) \quad \text { with } \mathrm{i}=1,2,3 \text { to } \mathrm{m}
\end{aligned}
$$

where sampling frequency, $f_{S}$, is equal to $1 / T_{S}$ and $m$ is the length of one data list.

The linearization allows for the use of least mean square regression for fitting the measured signals. In the hypothesis of well given $\tau_{\text {immobilized }}$ and $t_{0}$ values and an uncorrelated and Gaussian setup noise (verified with the set-up used and mainly limited by the used data acquisition system), an approximate confidence interval of level, $\alpha$, could be given by the $(\gamma=m-2)$ degrees of freedom Student Law, $t_{\alpha / 2}(\gamma)$, as

$$
\begin{aligned}
& \left|\kappa \hat{B}_{\text {immobilized }}\right|_{N_{i}}-\left.\kappa \hat{B}_{\text {immobilized }}\right|_{N_{i}} \mid \\
& \leq \frac{\sigma / \sqrt{N b}}{\sqrt{\sum_{i=1}^{m}\left(\tau\left(i T_{S}\right)-\frac{1}{m} \sum_{j=1}^{m} \tau\left(j T_{S}\right)\right)^{2}}} \cdot t_{(m-2) ; \alpha / 2}
\end{aligned}
$$

where $\left.\kappa \hat{B}_{\text {immobilized }}\right|_{N_{i}}$ is the estimated value of $\left.\kappa B_{\text {immobilized }}\right|_{N_{i}}, \sigma^{2}$ is the variance of the set-up magnetic noise and $N b$ is the number of measured signal (128 times).

Considering the confidence interval bars in the Fig. 7, firstly the noise variance for each sample was calculated from the relaxation measurements of the considered sample by subtraction of the fitted curve to the corresponding measured signal. Then, confidence intervals were evaluated with (4) for a $99 \%$ confidence interval (i.e. $t_{0.005}(m-2)=2.576$ with $\left.m=5000\right)$ and are given in Fig. 7 .

In the hypothesis of $\left.\kappa B_{\text {immobilized }}\right|_{N_{i}} \propto C_{N_{i}}^{\beta}$, where $C_{N_{i}}$ corresponds to $N_{i}$ sample concentration in $\mu \mathrm{mol}$ and $\beta$ is almost equal to 1 , and using previous Eq. (4), expected confidence interval of concentration evaluation is given, in the respect of highest concentration sample knowledge, by

$\left|\hat{C}_{N_{i}}-C_{N_{i}}\right| \approx \frac{C_{N_{1}}}{\kappa B_{\text {immobilized }} \mid N_{1}}\left|\kappa \hat{B}_{\text {immobilized }}\right| N_{i}-\kappa B_{\text {immobilized }}\left|N_{i}\right|$

We notice that for $1 / f$ noise, performed simulations lead to similar behavior of the confidence interval as a function of the variance of noise but no mathematical expression has been made so far.

\section{Discussion}

This paper relates the first magnetorelaxometry experiments performed with IGMRM. Discrepancy with the model ( $\beta \approx 0.5, c f$. Fig. 7) might be explained partially by the mechanical adjustment of the experimental set-up, the averaging and by the fast repetition time of the magnetic induction, $B_{a}$. Indeed, the $2.5 \mathrm{~s}$ duration without magnetic induction is not long enough for all MNPs to completely relax [12]. The confidence interval model proposed cannot be here used at best. Further measurements with longer relaxation period would be needed to get meaningful magnetorelaxometry results. Certainly, the experimental set-up used, identical to the one previously used for magnetorelaxometry experiments with GMI magnetometers [26] can be mechanically improved to enhanced magnetorelaxometry results.

Anyway, the high dynamic range, the high bandwidth and the high slew-rate of the IGMRMs allow for an improvement of measurements (high simple hardware gradiometer noise rejection efficiency, in open environment) without low temperature operation required for SQUID-based systems, as example. Compared to fluxgates, IGMRM should be less expensive and more compact. However, some optimization is still required to take advantage of their full potential. Differential measurements and signal acquisition taking benefit of the high slew-rate of the IGMRM 
magnetometer could lead to perform magnetorelaxometry experiments comparable to fluxgate ones. Especially, considering the linearized model proposed in (2), improvements in the confidence interval of the measurements could be obtained by early signals acquisition after magnetic induction decay. Indeed, early signals acquisition will increase the denominator of (4).

\section{Conclusion}

We have performed magnetorelaxometry experiments on superparamagnetic magnetite $\left(\mathrm{Fe}_{3} \mathrm{O}_{4}\right)$ nanoparticles using an IGMRM. The amplitudes extracted from fits were shown to be correlated to the concentration of the MNPs in samples. A model was proposed for the confidence interval of the amplitudes extracted from least mean square regression fits. The proposed model highlights ways of improvement as far as signal acquisition is concerned for the confidence of the extracted amplitude, $\left.\kappa B_{\text {immobilized }}\right|_{N_{i}}$. More, this formulation helps to compare magnetometer performances for magnetorelaxometry experiments. Further experiments will be performed taking into account experimental set-up improvements and measurement procedures.

\section{References}

[1] S.-B. Seo, J. Yang, W. Hyung, E.-J. Cho, T.-I. Lee, Y.J. Song, H.-G. Yoon, J.-S. Suh, Y.-M. Huh, S. Haam, Novel multifunctional PHDCA/PEI nano-drug carriers for simultaneous magnetically targeted cancer therapy and diagnosis via magnetic resonance imaging, Nanotechnology 18 (2007) 475105.

[2] L. Li, D. Chen, Y. Zhang, Z. Deng, X. Ren, X. Meng, F. Tang, J. Ren, L. Zhang, Magnetic and fluorescent multifunctional chitosan nanoparticles as smart drug delivery system, Nanotechnology 18 (2007) 405102.

[3] M. Tondra, A. Popple, A. Jander, R.L. Miller, N. Pekas, M.D. Porter, Microfabricated tools for manipulation and analysis of magnetic microcarriers, J. Magn. Magn. Mater. 293 (2005) 725.

[4] M. Kettering, J. Winter, M. Zeisberger, S. Bremer-Streck, H. Oehring, C. Bergemann, C. Alexiou, R. Hergt, K.J. Halbhuber, W.A. Kaiser, I. Hilger, Magnetic nanoparticles as bimodal tools in magnetically induced labelling and magnetic heating of tumour cells: an in vitro study, Nanotechnology 18 (2007) 175101.

[5] E. Parton, G. Borghs, R. De Palma, Biomedical applications using magnetic nanoparticles, Solid State Technol. 50 (8) (2007).

[6] W. Jia, G. Xu, R.J. Sclabassi, J.-G. Zhu, A. Bagic, M. Sun, Detection of magnetic nanoparticles with magnetoencephalography, J. Magn. Magn. Mater. 320 (2008) 1472-1478

[7] W. Weitschies, R. Kötitz, T. Bunte, L. Trahms, Determination of relaxing of remanent nanoparticle magnetization provides a novel binding-specific technique for the evaluation of immunoassays, Pharm. Pharmacol. Lett. 7 (1) (1997).

[8] F. Ludwig, S. Mäuselein, E. Heim, M. Schilling, Magnetorelaxometry of magnetic nanoparticles in magnetically unshielded environment utilizing a differential fluxgate arrangement, Rev. Sci. Instrum. 76 (2005) 106102.

[9] D. Eberbeck, Ch. Bergemann, S. Hartwig, U. Steinhoff, L. Trahms, Binding kinetics of magnetic nanoparticles on latex beads studied by magnetorelaxometry, Appl. Organometal. Chem. 18 (2004) 542-547.

[10] H.L. Grossman, W.R. Myers, V.J. Vreelan, R. Bruehl, M.D. Alper, C.R. Bertozzi, J Clarke, Detection of bacteria in suspension by using a superconducting quantum interference device, PNAS 101 (1)(2004) 129-134.

[11] R. Kotitz, L. Trahms, H. Koch, W. Weitschies, Biomagnetism: Fundamental Research and Clinical Applications, Elsevier Science Publishers, Amsterdam, 1995.

[12] F. Ludwig, E. Heim, D. Eberbeck, K. Schwarz, L. Trahms, M. Schilling, Comparison and calibration of fluxgate and SQUID magnetorelaxometry techniques for the characterization of magnetic core-shell nanoparticles, IEEE Trans. Magn. 45 (2009) 4857-4860.

[13] M.N. Baibich, J.M. Broto, A. Fert, F. Nguyen Van Dau, F. Petroff, P. Etienne, G. Creuzet, A. Friederich, J. Chazelas, Giant magnetoresistance of $(001) \mathrm{Fe} /(001) \mathrm{Cr}$ magnetic superlattices, Phys. Rev. Lett. 61 (21) (1988) 2472-2475.

[14] G. Binasch, P. Grünberg, F. Saurenbach, W. Zinn, Enhanced magnetoresistance in $\mathrm{Fe}-\mathrm{Cr}$ layered structures with antiferromagnetic interlayer exchange, Phys. Rev. B 39 (1989) 4282.
[15] D.R. Baselt, G.U. Lee, M. Natesan, S.W. Metzger, P.E. Sheehan, R.J. Colton, A biosensor based on magnetoresistance technology, Biosens. Bioelectron. 13 (1998) 731-739.

[16] J.C. Rife, M.M. Miller, P.E. Sheehan, C.R. Tamanaha, M. Tondra, L.J. Whitman, Design and performance of GMR sensors for the detection of magnetic microbeads in biosensors, Sens. Actuators A 107 (2003) 209-218.

[17] C. Carr, A.N. Matlachov, H. Sandin, M.A. Espy, R.H. Kraus Jr., Magnetic sensors for bioassay: HTS SQUIDs or GMRs? IEEE Trans. Appl. Supercond. 17 (2) (2007) 808-811.

[18] L. Perez, C. Dolabdjian, G. Waché, L. Butin, Advance in magnetoresistance magnetometer performances applied in eddy current sensor arrays, 16th WCNDT'04 Conference, 28 August-3 September, Montréal http://www.ndt. net/article/wcndt2004/pdf/eddy_current/296_dolabjian.pdf (2004).

[19] C. Dolabdjian, L. Perez, L. Butin, Magnetic field sensor and elementary module comprising the same, WO2006027459 (2006).

[20] E. Heim, S. Harling, F. Ludwig, H. Menzel, M. Schilling, Fluxgate magnetorelaxometry for characterization of hydrogel polymerization kinetics and physical entrapment capacity, J. Phys. Condens. Matter 20 (2008) 204106.

[21] D.V. Berkov, R. Kötitz, Irreversible relaxation behaviour of a general class of magnetic systems, J. Phys.: Condens. Matter 8 (1996) 1257-1266.

[22] F. Ludwig, E. Heim, S. Mauselein, D. Eberbeck, M. Schilling, Magnetorelaxometry of magnetic nanoparticles with fluxgate magnetometers for the analysis of biological targets, J. Magn. Magn. Mater. 293 (2005) 690-695.

[23] http://www.nve.com/glossary.php.

[24] C. Dolabdjian, A. Qasimi, D. Bloyet, Spatial resolution of SQUID magnetometers and comparison with low noise room temperature magnetic sensors, Physica C-Superconduct. Appl. 368 (1-4) (2002) 80-84.

[25] A. Nelder, R. Mead, A simplex method for function minimization, J. Comput. (USA) 7 (1965) 308.

[26] B. Dufay, S. Saez, M. Denoual, C. Dolabdjian, F. Ludwig, E. Heim, M. Schilling, L. Melo, A. Yelon, D. Menard, Magnetorelaxometry of nanoparticles using a GM magnetometer, Sens. Lett. 7 (3) (2009) 429-432.

\section{Biographies}

M. Denoual was born in 1975 and is an associate professor in the electronics team of the ENSICaen and University of Caen. He received his $\mathrm{PhD}$ degree in signal processing from the University of Rennes at Lannion in 2001 and MS degrees in electronics and microelectronics from the Polytechnic Institute of Toulouse and French Civil Aviation School at Toulouse. Before joining ENSICaen and University of Caen in 2005, Denoual was with the Fujita laboratory of the University of Tokyo, then with STMicroelectronics in Rennes and then with the BioMIS team of the ENS-Cachan where he conducted research on polymer and silicon microtechnologies for bioMEMS fabrication. His current research interests include bolometric detectors and applications of magnetism in biology and medicine.

S. Saez was born in Bayonne, France, in 1971. He received MS and PhD degrees in electronics and instrumentation from University of Caen, France, in 1995 and in 2000 , respectively.

He joined University of Caen in 2000 as assistant professor, during 5 years in the University Technological Institute of Alençon (IUT d'Alençon) and, now, in the Electronics Department. In the GREYC laboratory, his research interests include high sensitivity magnetometers (like GMI devices) and there applications (NDT and biomagnetism applications) in open or shielded environment.

F. Kauffman was born in Paris, France, on march 14, 1958. He received the BS degree in applied mathematics from Caen University and the MS degree in numerical analysis from Paris6 University. After he worked in the INRIA institute as a research scientist in digital processing applied to medicine. Since 1981 he is associate professor in Caen University. His main research area is applied statistics.

C. Dolabdjian was born in Enghien-les-Bains, France, in 1967. He received the MS and the PhD degree in electronics and instrumentation from the University of CAEN France, in 1991 and 1994, respectively, and the Habilitation Diploma in 2000.

In 1994, he joined the "Groupe de Recherche en Informatique, Image, automatique et Instrumentation de CAEN" CNRS UMR 6072 of ENSICAEN and University of CAEN as assistant professor, where he has been, professor of electronic, since 2001 . His research interests included studied, development, optimization, improvement and comparison of numerous very high sensitivity and very low magnetic noise sensors (SQUID, JFM, Flux-gate, GMR, GMI, $\mu$ Hall, Hybrid...), as well as their integration in applications, in open or close environment, like biomagnetism and Non-Destructive Testing

$\mathrm{He}$ is, also, director of the doctoral School "SIMEM" 2002-2009, head of the Electronic Team of the GREYC until 2007 and in charge of the "Licence Pro MCA" professional B.A. of the UCBN until 2001. 